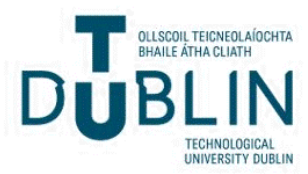

Technological University Dublin ARROW@TU Dublin

2014

\section{Doped quantum dots for photovoltaics}

\author{
Kevin Berwick \\ Technological University Dublin, kevin.berwick@tudublin.ie
}

A S. Baimuratov

Ivan D. Rukhlenko

Monash University Australia

See next page for additional authors

Follow this and additional works at: https://arrow.tudublin.ie/engscheceart

Part of the Engineering Commons

\section{Recommended Citation}

Baimuratov, A., Rukhlenko, I. \& Berwick, K. (2014). Doped quantum dots for photovoltaics. Light, Energy and the Environment, OSA Technical Digest (online) (Optical Society of America, 2014), paper JW6A.32. doi:org/10.1364/E2.2014.JW6A.32

This Article is brought to you for free and open access by the School of Electrical and Electronic Engineering at ARROW@TU Dublin. It has been accepted for inclusion in Articles by an authorized administrator of ARROW@TU Dublin. For more information, please contact arrow.admin@tudublin.ie, aisling.coyne@tudublin.ie, gerard.connolly@tudublin.ie.

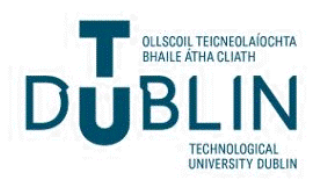




\section{Authors}

Kevin Berwick, A S. Baimuratov, Ivan D. Rukhlenko, Vidim Turkov, and A V. Fedorov 


\title{
Doped quantum dots for photovoltaics
}

\author{
Anvar S. Baimuratov, ${ }^{1,2, *}$ Ivan D. Rukhlenko, ${ }^{2,1}$ Vadim K. Turkov, ${ }^{1}$ \\ Irina O. Ponomareva, ${ }^{1}$ Mikhail Yu. Leonov, ${ }^{1}$ Tatiana S. Perova, ${ }^{3}$ \\ Kevin Berwick, ${ }^{4}$ Alexander V. Baranov, ${ }^{1}$ and Anatoly V. Fedorov ${ }^{1}$ \\ ${ }^{1}$ ITMO Univesity, 49 Kronverksky Avenue, 197101 Saint Petersburg, Russia \\ ${ }^{2}$ Monash University, Clayton Campus, Victoria 3800, Australia \\ ${ }^{3}$ Trinity College Dublin, The University of Dublin, Dublin 2, Ireland \\ ${ }^{4}$ Dublin Institute of Technology, Dublin 8, Ireland \\ baimuratov.anvar@gmail.com
}

\begin{abstract}
We report on a fundamental new property of level anticrossing of impurity states in semiconductor quantum dots, which can be used for fine tuning of the quantum dots' energy spectrum for photovoltaic applications.
\end{abstract}

OCIS codes: (160.4236) Nanomaterials; (250.5590) Quantum -well, -wire, and -dot devices.

\section{Introduction}

Colloidal semiconductor nanocrystals, also known as quantum dots, have unique optical and electronic properties that are controlled by their size, shape and chemical composition [1]. Doping of quantum dots with impurity atoms or ions is another method of tuning their physical properties, giving rise to many useful optical phenomena [2]. The presence of various impurities provides the basis for applications of quantum dots in nanodevices such as transistors [3], lightemitting diodes [4], bioimaging labels [5], chemical sensors [6], solar cells [7], and spintronic devices [8].

Since most of the optical properties of doped nanostructures strongly depend on their size, the study of the size dependencies of nanostructure impurity levels is a problem of both fundamental and applied significance. Precise, yet rather complex and time-consuming methods of single quantum-dot spectroscopy [9] allows one to resolve this problem experimentally. In this paper, we theoretically show that the simultaneous exposure of charge carriers to the Coulomb and confining potentials results in two different contributions to the size dependence of the quantum dot energy spectrum. The confining potential ceases to determine the structure of the spectrum with an increase of the quantum dot size while the Coulomb potential starts to dominate. As a consequence, the energies of some impurity states can accidentally coincide in quantum dot of a particular size. By examining the ordering of the energy levels for two limiting cases of small and large spherical quantum dots, we show that this accidental degeneracy is inherent to doped quantum dots regardless of their shape and composition. The degeneracy is removed by any interaction between the degenerate states, leading to the anticrossings in the size dependencies of the respective energy levels. Anticrossings engineering through varying the strength of the interaction enables one to precisely control the optical properties of quantum dots, as desired for photovoltaic applications.

\section{Results}

Consider a donor hydrogenic impurity in a spherical quantum dot of radius $R$. The impurity comprises a positive ion of charge $Z e$, which is located at the quantum dot center and coupled through the Coulomb interaction to an electron of charge $-e$ and effective mass $m_{e}$. By assuming that the quantum dot boundary is impenetrable to the electron, one can find the wave functions and energy spectrum of the impurity from the Schrödinger equation

$$
\left(-\frac{\hbar^{2}}{2 m_{e}} \nabla^{2}+V(\mathbf{r})\right) \Psi(\mathbf{r})=E \Psi(\mathbf{r})
$$

in which the confining potential is given by

$$
V(\mathbf{r})=\left\{\begin{array}{rr}
-\alpha / r, & r<R \\
\infty, & r \geq R
\end{array},\right.
$$

with $\alpha=Z e^{2} / \varepsilon_{0}$ and $\varepsilon_{0}$ being the low-frequency permittivity of the quantum dot. 

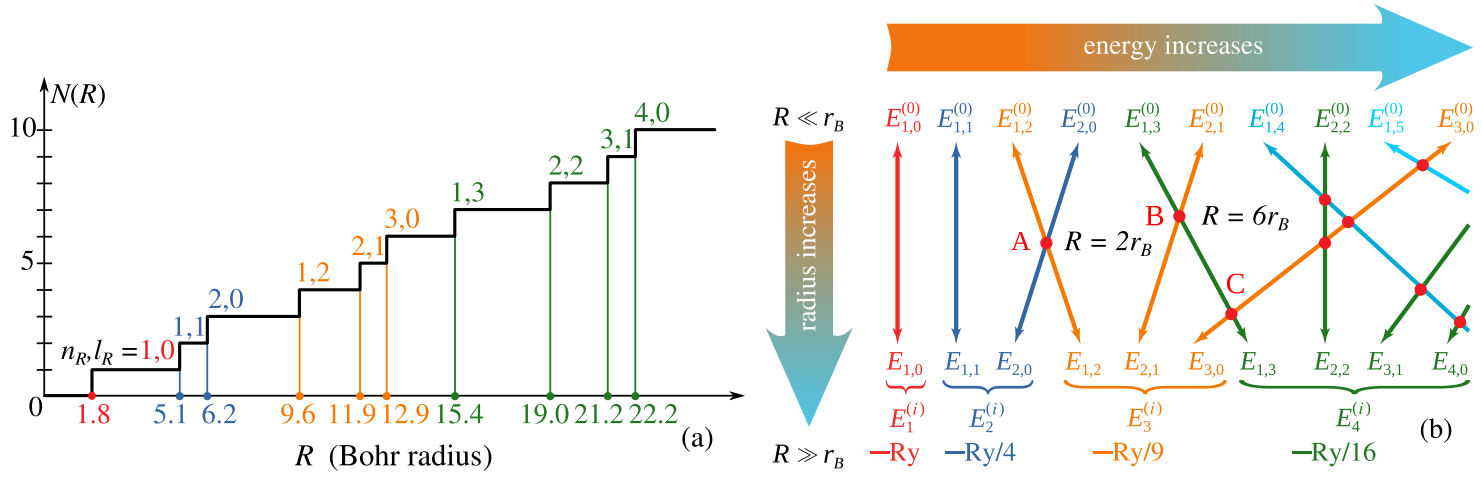

Fig. 1. (a) Number of negative-energy impurity states versus quantum dot radius $R$ and (b) variation of state ordering with $R$, illustrating formation of accidental-degeneracy points $\mathrm{A}, \mathrm{B}, \mathrm{C}$, etc. in the impurity energy spectrum.

Owing to the spherical symmetry of the confining potential, Eq. (1) allows an analytical solution [10], which enables a simple analysis of the absorption and photoluminescence spectra of quantum dots with donor impurities. It is possible, in particular, to estimate the quantum dot size by finding the number of absorption peaks lying below the fundamental absorption band. The peaks are centered at the energies of the lowest states in the quantum dot energy spectrum $E_{n, l}$, corresponding to different principal quantum numbers and angular momenta, $n=1,2,3, \ldots$ and $l=0,1,2, \ldots$. A more practical application is the problem of calculating the size of a quantum dot with a certain number of impurity states within the band gap.

To qualitatively understand how the energies and ordering of impurity states depend on the quantum dot size, we note that the impurity spectrum transforms into the spectrum of an infinitely high potential well without an impurity when $\alpha=0$, and becomes similar to the hydrogen-like spectrum for $R \gg r_{B}$, where $r_{B}=\hbar^{2} /\left(m_{e} \alpha\right)$ is the effective Bohr radius. The ordering of energy levels $E_{n, l}^{(0)}=\hbar^{2} \xi_{n, l}^{2} /\left(2 m_{e} R^{2}\right)$ in the first case is determined by the zeros $\xi_{n, l}$ of the spherical Bessel functions of the first kind $j_{l}(z)$. The hydrogen-like spectrum consists of negative- and positive-energy domains: the negative-energy spectrum $E_{v}^{(i)}=-\mathrm{Ry} / v^{2}(v=1,2,3, \ldots)$, where $\mathrm{Ry}=m_{e} \alpha^{2} /\left(2 \hbar^{2}\right)$ is the effective Rydberg, is discrete; and the positive-energy spectrum $E^{(i)}(\mathbf{k})=\operatorname{Ry} k^{2}(k>0)$ is continuous.

These limiting cases show that impurity centers should exhibit discrete energy spectra which may extend to negative energies. The negative-energy states are absent in small quantum dots, emerging one by one and growing in numbers with the quantum dot radius. The emergence is caused by the weakening of spatial confinement and the reduction of kinetic energy of the impurity electron, and its resulting distancing from the ion. The descend of quantum state $(n, l)$ from the positive-energy domain to the negative-energy domain occurs when its energy passes through zero in the quantum dot of radius

$$
R_{n, l}=\frac{\zeta_{n, 2 l+1}^{2}}{8} r_{B}
$$

where $\zeta_{n, 2 l+1}$ is the $n$th zero of the cylindrical Bessel function of the first kind $J_{2 l+1}(x)$.

To find the number of negative-energy impurity states in the quantum dot of radius $R$, one needs to determine the pair of quantum numbers $\left(n_{R}, l_{R}\right)$ such that $R<\min \left(R_{n_{R}, l_{R}}\right)$. Some algebra shows that there are

$$
N(R)=\frac{\left(n_{R}+l_{R}\right)\left(n_{R}+l_{R}+1\right)}{2}-l_{R}-1
$$

states of different energies within the interval $-\mathrm{Ry} \leq E_{n, l}<0$. These states are $(2 l+1)$-fold degenerate with respect to the angular momentum projections, making the total number of negative-energy sates equal to $\left(n_{R}+l_{R}\right)\left(n_{R}+l_{R}+\right.$ 1) $\left(2 n_{R}+2 l_{R}+1\right) / 6-\left(l_{R}+1\right)^{2}$. Figure 1(a) shows the staircase function $N(R)$, the steps of which correspond to new negative-energy levels $E_{n_{R}, l_{R}}$ successively emerging in the impurity spectrum. The figure enables one to estimate the quantum dot radius for a known number of negative-energy states, as well as to find the radius for a desired required number of states.

Comparison of the impurity spectrum with the spectrum of the quantum dot without an ion reveals essentially different orderings of their states. This is seen from the order of the first ten levels of the two spectra shown in Fig. 1(b) 
for $R>R_{4,0}$. By noticing that the ordering of levels $E_{n, l}^{(0)}$ coincides with that in small quantum dots (with $R \ll r_{B}$ ), and recalling that the impurity states form multiplets in large quantum dots (with $R \gg r_{B}$ ), we conclude that the energies of the orbitally nondegenerate impurity states will sometimes coincide as they shift with the quantum dot radius. Such an 'accidental' degeneracy occurs $e . g$. for a pair of states $(2,0)$ and $(1,2)$ in the quantum dot with $R=2 r_{B}$.

Accidental degeneracy resulting from different size dependencies of impurity states is removed by the interaction of the impurity with various excitations of the quantum dot (phonons, plasmons, excitons, etc.), external electromagnetic fields, or interaction with the environment. This causes splitting and anticrossing of the degenerate energy levels. The degree of degeneracy of the impurity states modified by the interaction is determined by the quantum numbers of the initial states and the nature of the interaction. One of the simplest level-anticrossing problems, arising for the lowest-energy accidentally degenerate states $(2,0)$ and $(1,2)$, requires solving the sixth-order secular equation. If an interaction of strength $V$ coupling these states does not couple the states of momentum projections $m=0, \pm 1$, and \pm 2 , then this equation has three different roots, two of which,

$$
\widetilde{E}_{ \pm}=\frac{1}{2}\left(E_{1,2}+E_{2,0} \pm \sqrt{\left(E_{1,2}-E_{2,0}\right)^{2}+4 V^{2}}\right)
$$

correspond to nondegenerate states, and one which corresponds to a four-fold degenerate state of energy $E_{1,2}$. Thus the interaction partially removes the six-fold degeneracy of the impurity states at point A. It should be noted that the degeneracy removal in real quantum dots is always complete due to the existence of coupling between the states of different momentum projections, splitting the four-fold degenerate state into four components.

\section{Conclusion}

We have shown that different size dependencies of impurity energy levels in quantum dots result in accidental level degeneracies, the removal of which leads to anticrossings in the size dependencies. The coupling of impurity states, which leads to the anticrossing, can be induced by interactions with elementary excitations residing inside the quantum dot or an external electromagnetic field. By controlling the kinds and strengths of the interactions, one can engineer the anticrossings and thus finely tune the optical properties of quantum dots for photovoltaic applications.

\section{References}

1. X. Wang, J. Zhuang, Q. Peng, and Y. Li, “A general strategy for nanocrystal synthesis," Nature 437, 121-124 (2005).

2. W.-k. Koh, A. Y. Koposov, J. T. Stewart, B. N. Pal, I. Robel, J. M. Pietryga, and V. I. Klimov, "Heavily doped n-type PbSe and PbS nanocrystals using ground-state charge transfer from cobaltocene," Sci. Rep. 3, 2004 (2013).

3. F. Hetsch, N. Zhao, S. V. Kershaw, and A. L. Rogach, "Quantum dot field effect transistors," Mater. Today 16, 312-325 (2013).

4. H. Menkara, R. A. Gilstrap, T. Morris, M. Minkara, B. K. Wagner, and C. J. Summers, "Development of nanophosphors for light emitting diodes," Opt. Express 19, A972-A981 (2011).

5. P. Wu and X.-P. Yan, "Doped quantum dots for chemo/biosensing and bioimaging," Chem. Soc. Rev. 42, 54895521 (2013).

6. R. Thakar, Y. Chen, and P. T. Snee, "Efficient emission from core/(doped) shell nanoparticles: Applications for chemical sensing," Nano Lett. 7, 3429-3432 (2007).

7. D. Zhitomirsky, M. Furukawa, J. Tang, P. Stadler, S. Hoogland, O. Voznyy, H. Liu, and E. H. Sargent, "N-type colloidal-quantum-dot solids for photovoltaics," Adv. Mater. 24, 6181-6185 (2012).

8. R. Beaulac, P. I. Archer, S. T. Ochsenbein, and D. R. Gamelin, "Mn-doped CdSe quantum dots: New inorganic materials for spin-electronics and spin-photonics," Adv. Funct. Mater. 18, 3873-3891 (2008).

9. S. Ates, S. M. Ulrich, A. Ulhaq, S. Reitzenstein, A. Löffler, S. Höfling, A. Forchel, and P. Michler, "Nonresonant dot-cavity coupling and its potential for resonant single-quantum-dot spectroscopy," Nature Photonics 3, 724-728 (2009).

10. L. Ma, J. Zhao, J. Wang, B. Wang, and G. Wang, "Magnetic properties of transition-metal impurities in silicon quantum dots," Phys. Rev. B 75, 045,312 (2007). 